\title{
The effectiveness of ultrasound-guided cervical transforaminal epidural steroid injections in cervical radiculopathy: a prospective pilot study
}

This article was published in the following Dove Medical Press journal: Journal of Pain Research

\author{
Xin Zhang ${ }^{1,2}$ \\ Haifeng Shi ${ }^{1,3}$ \\ Jin Zhou' \\ Yongming $\mathrm{Xu}^{\prime}$ \\ Shaofeng $\mathrm{Pu}^{\prime}$ \\ Yingying $L^{\prime}$ \\ Junzhen $\mathrm{Wu}^{\prime}$ \\ Yueping Cheng' \\ Dongping $\mathrm{Du}^{\prime}$ \\ 'Department of Anesthesiology, \\ Pain Management Center, Shanghai \\ Jiao Tong University Affiliated \\ Sixth People's Hospital, Shanghai, \\ China; ${ }^{2}$ Center for Translational \\ Pain Medicine, Department of \\ Anesthesiology, Duke University \\ School of Medicine, Durham, NC, \\ USA; ${ }^{3}$ Department of Anesthesiology, \\ Shanghai Fourth People's Hospital, \\ Shanghai, China
}

\begin{abstract}
Background: Cervical transforaminal epidural steroid injection (CTFESI) is used to provide pain relief and restore function in patients with cervical radiculopathy. Traditionally, it is performed under the guidance of fluoroscopy or computed tomography. Here, we introduce a novel technique - ultrasound-guided CTFESI - with which operators can easily distinguish the close soft tissue (nerve, vessels) around the cervical foramina to avoid intravascular injection during the procedure.
\end{abstract}

Objective: To present the immediate and long-term effectiveness of ultrasound-guided CTFESI in patients with cervical radiculopathy in an academic pain-management center with prospective clinic experiments.

Methods: Fifteen patients with cervical radiculopathy who were resistant to conservative therapies and ultrasound-guided selective cervical spinal nerve-root injections, were treated with ultrasound-guided CTFESI. During the injection procedures, the needle tips were reconfirmed by real-time fluoroscopy. Pain numeric rating-scale and neck-disability-index scores were assessed from onset to six months after the procedures.

Results: During the procedures, based on real-time fluoroscopic confirmation, the injection solution outlined the spinal nerve root and spread into the epidural space in most cases (14 of 15). All patients reported pain relief within 10 minutes after the injection. The majority of patients (eleven of 15) experienced pain relief and neck-disability index-score improvement throughout the 6-month study period. No patient experienced any complication.

Conclusion: We suggest that ultrasound-guided CTFESI is an effective, safe, and simple procedure free of radiation or magnetization and provides sustained pain relief in patients with cervical radiculopathy who have failed previous conservation therapies.

Keywords: cervical radiculopathy, ultrasound-guided, cervical transforaminal epidural steroid injection, steroid

\section{Introduction}

Cervical radiculopathy is a common condition leading to radicular pain, which affects approximately one per 1,000 people per year. ${ }^{1}$ Irritation of the cervical spinal nerve roots (NRs) by lateral disk herniation or foraminal stenosis is usually considered the main cause. Although its natural history can be favorable, not all patients recover naturally. Many remain with severe pain and require further treatment. ${ }^{2}$

Cervical epidural steroid injection is a common interventional treatment for cervical radicular pain, ${ }^{3}$ particularly in those patients who are resistant to conservation therapies. Usually, two methods are employed for cervical epidural steroid delivery: an
Correspondence: Xin Zhang; Dongping Du

Pain Management Center, Shanghai Jiao Tong University Affiliated Sixth People's Hospital, 600 Yishan Road, Shanghai 200235, China

Tel +862124058896

Fax +862124058330

Email xin.zhang3@duke.edu;

dudp@sjtu.edu.cn 
interlaminar approach and a transforaminal approach. Compared to these two methods, the cervical transforaminal epidural steroid injection (CTFESI) approach can ensure direct delivery of steroids into the anterior epidural space, which is closer to the irritated spinal NRs or the responsive cervical intervertebral disk, causing it to be more advantageous. However, during CTFESI procedures, inadvertent intraarterial injection can occur, resulting in some rare but severe complications, such as cerebellar or spinal cord infarction. ${ }^{4}$ Usually, CTFESI is performed under fluoroscopic guidance. As fluoroscopy cannot verify the soft tissue or nerve tissue close to the foramina, physicians perform CTFESI carefully using real-time fluoroscopy with contrast. Understandably, the risk of serious neurological complications is still not zero. ${ }^{5}$

Ultrasound may have more advantages than fluoroscopy, owing to its high identification of soft tissue, nerves, vessels, and the spread of injected drugs around the nerve. In 2009, Narouze et al introduced a convenient procedure called the ultrasound-guided cervical selective NR (SNR) injection. ${ }^{6}$ This approach facilitates the identification of critical vessels at unexpected locations relative to the cervical foramina and avoids injury to such vessels. In our pain-management center, we frequently choose the SNR approach as routine treatment for cervical radicular pain. With this approach, drug delivery occurs quite closely to the irritated spinal NRs, and yet in most cases these drugs still cannot spread directly into the epidural space. To address this problem, we introduce a novel approach: ultrasound-guided CTFESI. Using this technique, operators can precisely inject the drug into the epidural space through the cervical foramina. We also assess the effectiveness of this approach.

\section{Methods}

\section{Subjects}

The study protocol was carried out in accordance with the principles of the Declaration of Helsinki, and was reviewed and approved by the institutional review board of Shanghai Jiao Tong University Affiliated Shanghai Sixth People's Hospital. All patients agreed to the study and provided written consent to participate and have procedural images published. From May 2014 to April 2016, we recruited a total of 528 patients with cervical radiculopathy who were suspected of having cervical radicular pain based on physical examination. Cervical computed tomography and magnetic resonance imaging in the corresponding NR location confirmed herniated cervical intervertebral disk and spinal stenosis. A total of 88 of these patients showed limited responses to 4-week conservative therapies (nonsteroidal anti-inflammation drugs or analgesics, with/without physical therapy). For this study and further invasive procedures, the following exclusion criteria were applied: patients with known allergies to contrast dye or local anesthetics, pregnant, tumor or tumor metastasis in the involved area of the spine, spinal instability, infectious spinal disease, or active coagulopathy. We treated these 88 patients with two consecutive ultrasound-guided cervical SNR injections (once every 2 weeks, $2.5 \mathrm{mg}$ of $\beta$-methasone each time; Schering-Plow, Kenilworth, NJ, USA). The majority of them experienced pain relief after SNR. However, 15 of these patients (eight men, seven women) still did not show obvious improvements (NRS >7). Finally, we performed a single ultrasound-guided CTFESI ( $5 \mathrm{mg}$ dexamethasone) for these 15 patients (Figure 1).

\section{Procedure}

CTFESI was performed by an experienced physician (XZ) in the Pain Management Center of Shanghai Jiao Tong University Affiliated Sixth People's Hospital and took place in the procedure suite under ultrasound and $\mathrm{C}$-arm fluoroscopic guidance. Briefly, patients were placed in the lateral decubitus position. The physician stood in front of the patient's face. After preparation of the skin with antiseptic solution, a linear high-frequency ultrasound probe $(6 \mathrm{MHz}, \mathrm{S}-\mathrm{Nerve}$; SonoSite, Bothell, WA, USA) was placed transversely on the lateral side of the neck to obtain a transverse axial view. The cervical spinal nerve can be identified between the posterior and anterior tubercle in this view (Figure 2A). Then, the probe was rotated vertically to gain a longitudinal view of the spinal nerve: this nerve running through the cervical foramina can be obtained in this image. Further, deep inside the foramina, the epidural membrane was visible as a hyperechoic line (Figure 2B). Further, with the cervical foramina as the clockwise axis, the probe was rotated until it was parallel to the sternocleidomastoid muscle. Here, the spinal nerve is seen only as a curvature above the cervical foramina (Figure $2 \mathrm{C}$ ). The puncture needle can then be advanced easily toward the cervical foramina from the anterior to posterior direction using the ultrasound-guided in-plane approach. The needle tip was advanced until it hit the foraminal edge (Figure 2D). After careful aspiration, $1 \mathrm{~mL} 0.9 \% \mathrm{NaCl}$ was injected to confirm that the needle tip was in the proper position. After confirmation, $2 \mathrm{~mL}$ contrast medium was injected under real-time fluoroscopic guidance. No intravascular injections were observed in any of the procedures. Fluoroscopic images were captured 2 minutes after contrast-medium injection, showing the medium outlining the cervical spinal nerve-end root and spreading into the epidural space without any other 
A

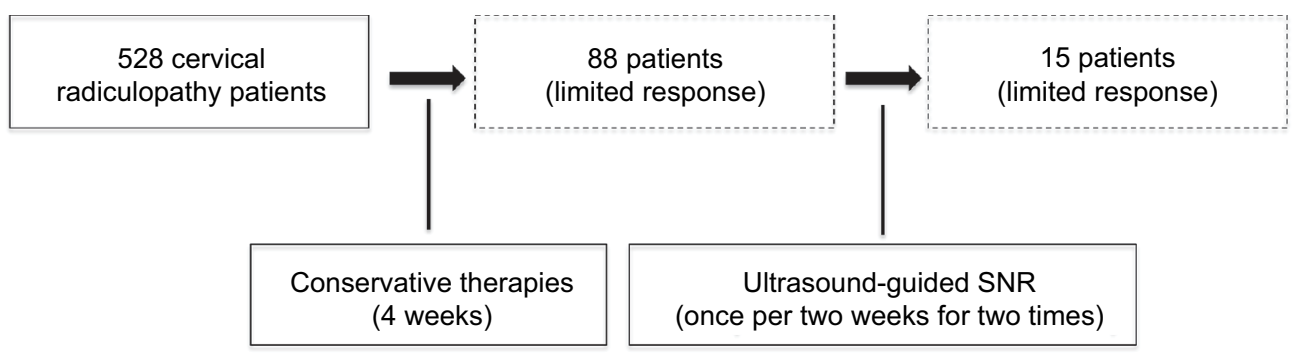

- - - - Limited response

B

\begin{tabular}{|c|c|c|c|c|c|}
\hline Case\# & Sex & Age (years) & Irritation segments & Sides & Duration (months) \\
\hline 1 & $\mathrm{~F}$ & 57 & C5 & $\mathrm{L}$ & 15 \\
\hline 2 & $M$ & 45 & C6 & $\mathrm{L}$ & 8 \\
\hline 3 & $M$ & 48 & C5 & $\mathrm{L}$ & 11 \\
\hline 4 & $\mathrm{~F}$ & 51 & $\mathrm{C7}$ & $\mathrm{R}$ & 9 \\
\hline 5 & $M$ & 47 & $\mathrm{C7}$ & $\mathrm{R}$ & 8 \\
\hline 6 & $\mathrm{~F}$ & 45 & C7 & $\mathrm{L}$ & 14 \\
\hline 7 & $\mathrm{~F}$ & 32 & C6 & $\mathrm{L}$ & 15 \\
\hline 8 & $M$ & 62 & C6 & $\mathrm{R}$ & 15 \\
\hline 9 & $M$ & 54 & $\mathrm{C7}$ & $\mathrm{R}$ & 12 \\
\hline 10 & $M$ & 48 & C6 & $\mathrm{L}$ & 15 \\
\hline 11 & $M$ & 45 & C6 & $\mathrm{R}$ & 11 \\
\hline 12 & $\mathrm{~F}$ & 38 & C6 & $\mathrm{R}$ & 10 \\
\hline 13 & $\mathrm{~F}$ & 26 & C5 & $\mathrm{R}$ & 9 \\
\hline 14 & $M$ & 28 & C7 & $\mathrm{L}$ & 9 \\
\hline 15 & $\mathrm{~F}$ & 33 & C5 & $\mathrm{R}$ & 8 \\
\hline
\end{tabular}

Figure I (A) Study protocol; (B) patient characteristics.

Abbreviation: SNR, selective nerve root.

intravascular injection (Figure 3A and B). After real-time fluoroscopic and ultrasound-based confirmation that no inadvertent intravascular injection had occurred, $1 \mathrm{~mL}$ of $5 \mathrm{mg} / \mathrm{mL}$ dexamethasone and $1 \mathrm{~mL} 0.5 \%$ bupivacaine was injected under ultrasound guidance.

\section{Data recording}

During the CTFESI procedure, heart rate, blood pressure, $\mathrm{SpO}_{2}$, and all other symptoms were recorded. Patients were asked to score their pain using a numerical rating scale (NRS; 10 maximum and 0 minimum) prior to the injection, and at 10 minutes, 2 weeks, 3 months, and 6 months postinjection. Neck-disability index (NDI) questionnaires that suggested interpreting the scores as per the following ranges were also used to assess neck function prior to the injection and at 3 and 6 months postinjection: $\leq 4$, no disability; $5-14$, mild disability; $15-24$, moderate disability; $25-34$, severe disability; $\geq 35$, complete disability. When NRS and NDI scores had improved by $>50 \%$ and $>40 \%$, respectively, we considered the treatment effect successful. ${ }^{7}$

\section{Statistical analysis}

Data are presented as mean \pm SD. NRS and NDI over time were analyzed by one-way ANOVA with nonparametric tests. Statistical significance was defined as $P<0.05$. All statistical analyses were performed using GraphPad Prism 7 (GraphPad Software, San Diego, CA, USA).

\section{Results}

Subject characteristics are presented in Figure 1B. Fifteen patients (median age 45 [26-62] years, eight male and seven female) received CTFESI. The mean symptom duration was $11.2(8-15)$ months. The mean preinjection NRS was 7.6 \pm 0.74 . 


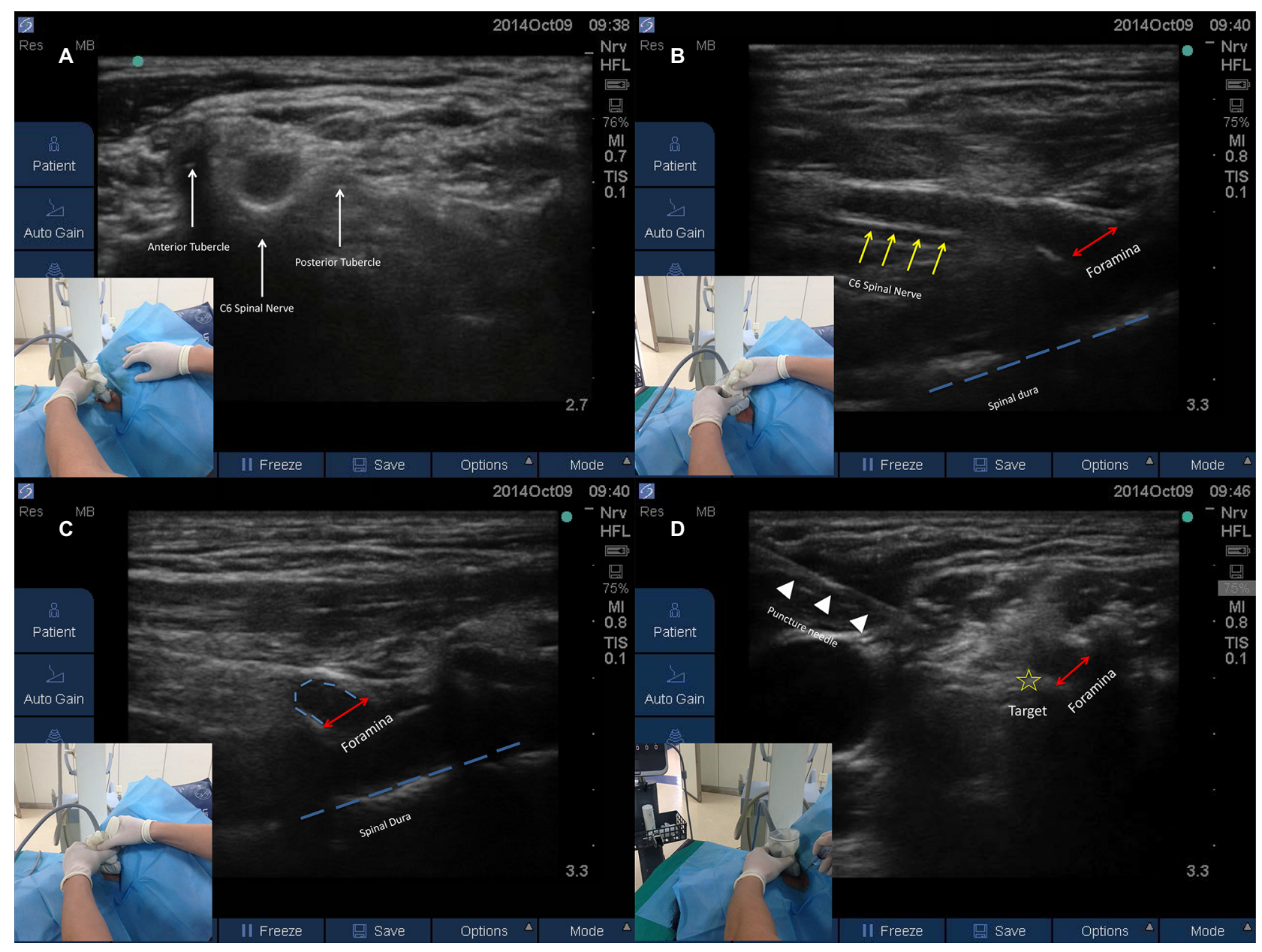

Figure 2 Illustration of ultrasound-guided CTFESI.

Notes: Point-to-point transducer positions for each step. The patient was facing the physician, with the head seen at left in the images. Red lines show the foramina, the yellow star shows the target of the needle tip, and the blue line shows the spinal dura.

Abbreviation: CTFESI, cervical transforaminal epidural steroid injection.

Ten minutes after the injection, all patients reported pain relief, and mean NRS score was recorded as $1.06 \pm 0.70$. Pain scores were low throughout the observation period ( 2 weeks, $2.4 \pm 1.99, P<0.01$ vs NRS at baseline; 3 months, $2.53 \pm 2.44$, $P<0.01$ vs NRS at baseline; 6 months, $3.13 \pm 2.20, P<0.05$, vs NRS at baseline). The mean preinjection NDI was $22.4 \pm 3.33$. In addition, scores also decreased significantly after CTFESI (3 months, $15.4 \pm 4.67 ; P<0.01$ vs NDI at baseline; 6 months, $12.87 \pm 6.62, P<0.01$ vs NDI at baseline; Figure $4 \mathrm{~A}$ ).

We considered an improvement in NRS and NDI scores by $>50 \%$ and $>40 \%$, respectively, as successful treatment. ${ }^{7}$ Success rates of CTFESI at 3 and 6 months were $60 \%$ and $73.33 \%$, respectively (Figure 4B). Four of the 15 patients still reported severe cervical radicular pain and limited improvement with respect to neck-disability degree at 6 months postinjection, and they were recommended to undergo surgery. In most cases (14 of 15), fluoroscopic images showed that the contrast medium not only outlined the ventral branch of the cervical spinal nerve-end root but also spread into the epidural space, which indicated successful epidural injections. Such complications as bleeding, epidural puncture, and intravascular injection did not occur in any procedures.

\section{Discussion}

With the development of high-resolution ultrasound, ultrasound-guided cervical injections have been used widely in the clinic. Ultrasound-guided cervical medial branch blocks provide significant improvement in managing chronic neck pain of cervical facet-joint origin. ${ }^{8}$ Furthermore, the ultrasound-guided superficial cervical plexus block procedure can be used for patients who are scheduled to undergo carotid endarterectomy and superficial neck surgery or those with ear and neck pain caused by entrapment of the superficial cervical plexus. ${ }^{9}$ However, in cases of cervical radiculopathy with a 


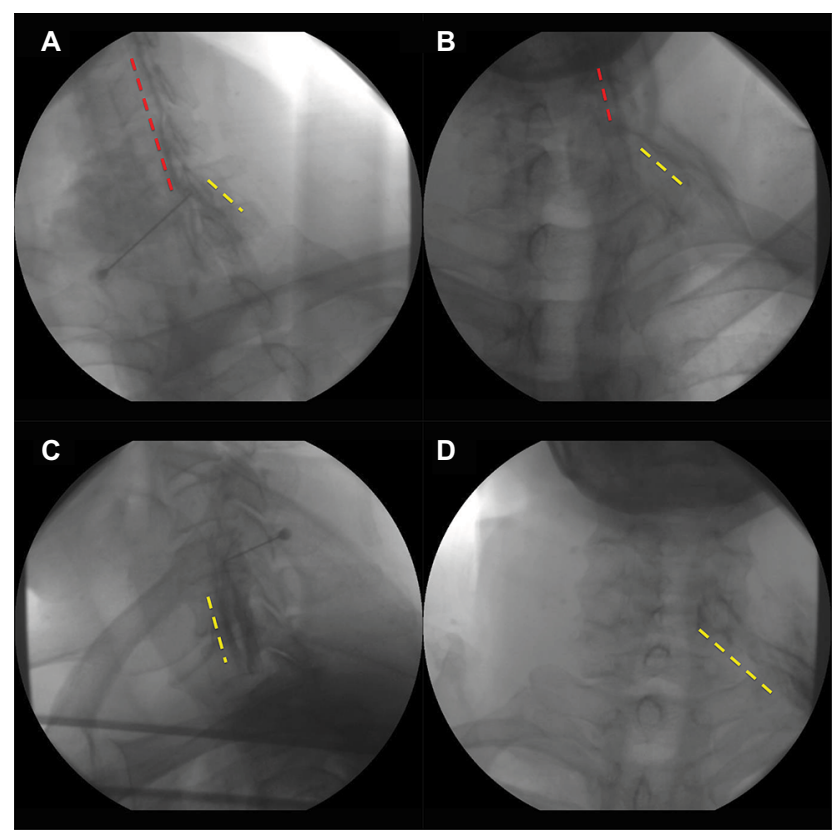

Figure 3 (A) Oblique view for C6 ultrasound-guided CTFESI under fluoroscopy; (B), anteroposterior view for C6 ultrasound-guided CTFESI under fluoroscopy; (C), oblique view for C6 ultrasound-guided SNR under fluoroscopy; (D): anteroposterior view for C6 ultrasound-guided SNR under fluoroscopy. Yellow dotted lines, contrast medium surrounding the spinal nerve; red dotted lines, contrast medium in epidural space.

Abbreviation: CTFESI, cervical transforaminal epidural steroid injection; SNR selective nerve root. herniated disk or osseous foraminal encroachment, treatment directly targeting a selective cervical root block, as well as relevant cervical epidural space, still acts as the first choice in the clinic..$^{10}$ SNR injection, which was introduced in 2009 , is a routine procedure for cervical radiculopathy patients. ${ }^{6}$ Jee et al also suggested that ultrasound-guided SNR might facilitate identification of critical vessels at unexpected locations relative to the intervertebral foramen and avoid injury to such vessels. ${ }^{11}$ However, there is still a physical distance from the spinal NR to the cervical foramina (Figure $3 \mathrm{C}$ and $\mathrm{D}$ ), and ultrasoundguided SNR injection still cannot completely replace epidural injection. Even using $4 \mathrm{~mL}$ volume solution, only $24.5 \%$ of the SNR injection can deliver the drug into the epidural space. ${ }^{12}$

In this study, 528 cervical radiculopathy patients were recruited, and the majority ( 440 of 528) gained pain relief after 4 weeks of conservative therapy. The remaining 88 patients received ultrasound-guided SNR injections twice in one month. Around $17 \%$ of these patients ( 15 of 88 ) still showed limited symptom resolution. We treated these 15 patients with ultrasound-guided CTFESI to determine whether CTFESI could provide patients with further pain relief. Remarkably, eleven of these 15 patients reported significant pain relief

A

\begin{tabular}{|c|c|c|c|c|c|}
\hline & Baseline & 10 minutes & 2 weeks & 3 months & 6 months \\
\hline $\begin{array}{c}\text { Numeric rating scale } \\
\text { (NRS) }\end{array}$ & $7.6 \pm 22.4$ & $7.6 \pm 0.7^{* *}$ & $2.4 \pm 1.99^{* *}$ & $2.53 \pm 2.44^{* *}$ & $3.13 \pm 2.20^{* *}$ \\
\hline $\begin{array}{l}\text { Neck disability index } \\
\text { (NDI) }\end{array}$ & $22.4 \pm 3.33$ & & & $15.4 \pm 4.67 \#$ & $12.8 \pm 6.62^{\#}$ \\
\hline
\end{tabular}

B

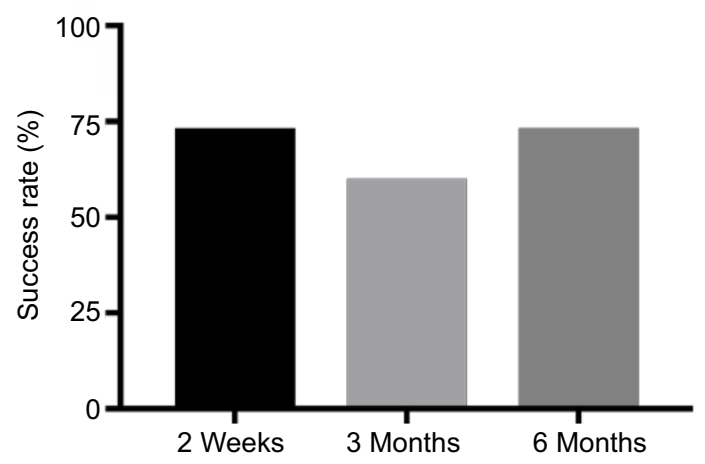

Figure 4 (A) The assessment of immediate and long-term pain relief and neck-function restoration after CTFESI; (B) success rate of CTFESI at different time points. 
6 months after the injection. These findings suggested that ultrasound-guided CTFESI could result in better benefits to patients who are resistant to conservative therapies and SNR.

In the ultrasound-guided CTFESI procedure, we first captured a longitudinal image of the cervical spinal nerve to obtain the whole view, wherein we could see the spinal nerve running through the foramina. We also measured the distance from the foramina to the epidural membrane in this image, which typically measured around $5 \mathrm{~mm}$. This provided sufficient space to perform the injection safely. On this longitudinal view, the spinal nerve and cervical foramina was able to be clearly identified; however, because of the obstruction from the patient's shoulder, we could not advance the injection needle easily in this posture. As such, we rotated the probe clockwise to make more space. As the probe was roughly parallel to the sternocleidomastoid muscle, the foramina was seen only as a curvature. At this posture, the needle tip was able to be advanced precisely toward the cervical foramina from the anterior to posterior direction with an ultrasound-guided in-plane approach. Once the needle tip hit the foramina edge, we injected $1 \mathrm{~mL}$ $0.9 \% \mathrm{NaCl}$ to confirm the tip location at first, and then used contrast medium to reconfirm the needle tip's location under real-time fluoroscopic guidance. In most cases, we saw the solution spreading into the epidural space, which indicated successful epidural injection. It was difficult to detect the needle tip once it entered the cervical foramina, so we only let the needle hit the foraminal edge, instead of advancing further. Although we believe ultrasound-guided CTFESI is a simple and safe procedure, we still highly recommend careful evaluation of vulnerable vessels around the cervical NR with ultrasound before the procedure. ${ }^{13}$ As CTFESI can be performed in the lateral decubitus position, this technique can also benefit those patients who can lie in the prone position, the routine request for fluorescence-guided procedures.

\section{Conclusion}

In the present study, ultrasound-guided CTFESI showed significant clinical effectiveness in patients resistant to classic ultrasound-guided SNR. Therefore, we suggest that ultrasound-guided CTFESI is an effective, safe, and simple procedure free of radiation or magnetization and provides sustained pain relief in patients with cervical radiculopathy who have failed previous conservation therapies.

\section{Acknowledgments}

This work was supported in part by the General Program of National Natural Science Foundation of China (81370933,
81400803, and 81672237). This work was supported in part by the National Natural Science Foundation of China (81400803 to XZ, 8167237 and 81370093, to DPD).

\section{Author contributions}

$\mathrm{DD}$ and $\mathrm{XZ}$ were responsible for study design and supervised the whole study. JZ, YX, SP, XZ, DD, and YL recruited all the patients. HS, XZ, and YC performed the ultrasound-guided CTFESI. JW and YC performed the ultrasound-guided SNR. $\mathrm{DD}$ and $\mathrm{XZ}$ obtained funding and provided administrative support. XZ drafted the manuscript. All authors contributed to data analysis, drafting and revising the article, gave final approval of the version to be published, and agree to be accountable for all aspects of the work.

\section{Disclosure}

The authors report no conflicts of interest in this work.

\section{References}

1. Radhakrishnan K, Litchy WJ, O'Fallon WM, Kurland LT. Epidemiology of cervical radiculopathy. A population-based study from Rochester, Minnesota, 1976 through 1990. Brain. 1994;117(Pt 2):325-335.

2. Rathmell JP, Aprill C, Bogduk N. Cervical transforaminal injection of steroids. Anesthesiology. 2004;100(6):1595-1600.

3. House LM, Barrette K, Mattie R, Mccormick ZL. Cervical epidural steroid injection: techniques and evidence. Phys Med Rehabil Clin N Am. 2018;29(1):1-17.

4. Schneider BJ, Maybin S, Sturos E. Safety and complications of cervical epidural steroid injections. Phys Med Rehabil Clin N Am. 2018;29(1):155-169.

5. El-Yahchouchi CA, Plastaras CT, Maus TP, et al. Adverse event rates associated with transforaminal and interlaminar epidural steroid injections: a multi-institutional study. Pain Med. 2016;17(2):239-249.

6. Narouze SN, Vydyanathan A, Kapural L, Sessler DI, Mekhail N. Ultrasound-guided cervical selective nerve root block: a fluoroscopycontrolled feasibility study. Reg Anesth Pain Med. 2009;34(4):343-348.

7. Manchikanti L, Singh V, Falco FJ, Cash KA, Pampati V. Lumbar facet joint nerve blocks in managing chronic facet joint pain: one-year follow-up of a randomized, double-blind controlled trial: Clinical Trial NCT00355914. Pain Physician. 2008;11(2):121-132.

8. Manchikanti L, Singh V, Falco FJ, Cash KA, Fellows B. Comparative outcomes of a 2-year follow-up of cervical medial branch blocks in management of chronic neck pain: a randomized, double-blind controlled trial. Pain Physician. 2010;13(5):437-450.

9. Pandit JJ, Satya-Krishna R, Gration P. Superficial or deep cervical plexus block for carotid endarterectomy: a systematic review of complications. Br J Anaesth. 2007;99(2):159-169.

10. Huston CW, Slipman CW. Diagnostic selective nerve root blocks: indications and usefulness. Phys Med Rehabil Clin NAm. 2002;13(3):545-565.

11. Jee H, Lee JH, Kim J, Park KD, Lee WY, Park Y. Ultrasound-guided selective nerve root block versus fluoroscopy-guided transforaminal block for the treatment of radicular pain in the lower cervical spine: a randomized, blinded, controlled study. Skeletal Radiol. 2013;42(1):69-78.

12. Kang S, Yang SN, Kim SH, Byun CW, Yoon JS. Ultrasound-guided cervical nerve root block: does volume affect the spreading pattern? Pain Med. 2016;17(11):1978-1984.

13. Park D. Distribution patterns of the vulnerable vessels around cervical nerve roots: a computed tomography-based study. Am J Phys Med Rehabil. 2018;97(4):242-247. 
The Journal of Pain Research is an international, peer reviewed, open access, online journal that welcomes laboratory and clinical findings in the fields of pain research and the prevention and management of pain. Original research, reviews, symposium reports, hypothesis formation and commentaries are all considered for publication
The manuscript management system is completely online and includes a very quick and fair peer-review system, which is all easy to use. Visit http://www.dovepress.com/testimonials.php to read real quotes from published authors.

Submit your manuscript here: https://www.dovepress.com/journal-of-pain-research-journal 